\title{
Kinase-driven metabolic signalling as a predictor of response to carboplatin- paclitaxel adjuvant treatment in advanced ovarian cancers
}

Maria Isabella Sereni ${ }^{1,2,3,6}$, Elisa Baldelli ${ }^{1,6}$, Guido Gambara ${ }^{1}$, Antonella Ravaggi ${ }^{4}, \mathrm{~K}$ Alex Hodge ${ }^{1}$, David S Alberts ${ }^{5}$, Jose M Guillen-Rodriguez ${ }^{5}$, Ting Dong ${ }^{1}$, Maurizio Memo ${ }^{2}$, Franco Odicino ${ }^{4}$, Roberto Angioli ${ }^{3}$, Lance A Liotta ${ }^{1}$, Sergio L Pecorelli ${ }^{4}$, Emanuel F Petricoin ${ }^{1}$ and Mariaelena Pierobon ${ }^{*}, 1$

${ }^{1}$ Center for Applied Proteomics and Molecular Medicine, George Mason University, 10920 George Mason Circle, Manassas, VA 20110, USA; ${ }^{2}$ Department of Molecular and Translational Medicine, University of Brescia, Viale Europa 11, 25123 Brescia, Italy; ${ }^{3}$ Department of Obstetrics and Gynecology, Campus Bio-Medico University of Rome, Via Alvaro del Portillo 21, 00128 Roma, Italy; ${ }^{4}$ Division of Gynecologic Oncology, 'Angelo Nocivelli' Institute of Molecular Medicine, University of Brescia, Piazzale Spedali Civili 1, 25123 Brescia, Italy and ${ }^{5}$ The University of Arizona Cancer Center, 3838N Campbell Ave, Tucson, AZ 85719, USA

Background: The biological mechanisms underlying early- and advanced-stage epithelial ovarian cancers (EOCs) are still poorly understood. This study explored kinase-driven metabolic signalling in early and advanced EOCs, and its role in tumour progression and response to carboplatin-paclitaxel treatment.

Methods: Tumour epithelia were isolated from two independent sets of primary EOC ( $n=72$ and 30 for the discovery and the validation sets, respectively) via laser capture microdissection. Reverse phase protein microarrays were used to broadly profile the kinase-driven metabolic signalling of EOC with particular emphasis on the LBK1-AMPK and AKT-mTOR axes. Signalling activation was compared between early and advanced lesions, and carboplatin-paclitaxel-sensitive and -resistant tumours.

Results: Advanced EOCs were characterised by a heterogeneous kinase-driven metabolic signature and decreased phosphorylation of the AMPK-AKT-mTOR axis compared to early EOC $(P<0.05$ for AMPK $\alpha$ T172, AMPK $\alpha 1$ S485, AMPK $\beta 1$ S108, AKT S473 and T308, mTOR S2448, p70S6 S371, 4EBP1 S65, GSK-3 $\alpha / \beta$ S21/9, FOXO1 T24/FOXO3 T32, and FOXO1 S256). Advanced tumours with low relative activation of the metabolic signature and increased FOXO1 T24/FOXO3 T32 phosphorylation $(P=0.041)$ were associated with carboplatin-paclitaxel resistance.

Conclusions: If validated in a larger cohort of patients, the decreased AMPK-AKT-mTOR activation and phosphorylation of FOXO1 T24/FOXO3 T32 may help identify carboplatin-paclitaxel-resistant EOC patients.

Epithelial ovarian cancer (EOC) is the second most common gynaecological malignancy and it represents the fifth leading cause of cancer-related deaths in women (Siegel et al, 2016). Owing to the absence of specific symptoms at the onset of the disease and the lack of effective screening programmes, most patients receive medical attention when the disease is already spread outside the

\footnotetext{
*Correspondence: Dr M Pierobon; E-mail: mpierobo@gmu.edu

${ }^{6}$ These authors contributed equally to this work.
}

Received 31 January 2017; revised 19 May 2017; accepted 1 June 2017; published online 29 June 2017

(C) 2017 Cancer Research UK. All rights reserved 0007 - 0920/17 
pelvis (Zeppernick and Meinhold-Heerlein, 2014). For these reasons, the prognosis for EOC is still poor, with a 5-year overall survival rate between 14 and $37 \%$ for women diagnosed with locally or systemically advanced disease (Rosendahl et al, 2016). The development of more effective therapeutic approaches for ovarian cancer patients has proven to be extremely challenging because EOC is a heterogeneous disease and the molecular events driving ovarian carcinogenesis are relatively unknown (Kurman and Shih, 2010). For these reasons, surgical excision of the tumour and platinum-based \pm paclitaxel treatment remains the best therapeutic option for EOC even in an era where targeted treatments are already considered standard of care for other tumour types.

It has been postulated that early- and advanced-stage EOC may constitute distinct conditions each characterised by specific origins, pathogenesis, and clinical history (Kurman and Shih, 2010, 2011). For example, it is now known that high-grade serous ovarian cancers originate from the fallopian tubes while other EOC histotypes develop directly from the epithelial surface (Reade et al, 2014). As a consequence, the site of origin may directly influence the molecular landscape of the different EOC subtypes. In addition, while early-stage tumours can be of larger size at diagnosis compared to advanced lesions, they also tend to be contained within the pelvis and have less aggressive behaviour (Groen et al, 2015). Therefore, understanding the molecular mechanisms underlying different stages of the disease may lead to the identification of predictive and therapeutic biomarkers for personalised medicine of EOC. In this context, while it has been previously shown that ovarian cancer is among the most biologically complex of all solid tumours (explaining to some degree the lack of successful molecularly targeted agents and early development of drug resistance) (Taetle et al, 1999; Nelson et al, 2000), a recent analysis suggested that treatment selection based on molecular profiling can both identify patients with less favourable prognosis and positively affect EOC patients' overall survival by leading to the selection of individualised treatments matched to the patient-specific molecular profile (Herzog et al, 2016).

Metabolic reprogramming is considered an important hallmark in EOC, and the use of metformin, a metabolic modulator commonly used to treat type II diabetes, has shown promising results in ovarian cancer (Hanahan and Weinberg, 2011; Dilokthornsakul et al, 2013; Kumar et al, 2013). Metformin antitumour effects are exerted through the activation of the AMPactivated protein kinase (AMPK), a cellular energy sensor that regulates metabolic functions by modulating kinase-dependent signalling cascades (Irie et al, 2016). The crosstalk between the LKB1-AMPK and the AKT-mTOR pathways regulates a significant portion of the cellular bioenergetic capacity under physiological conditions and derangements of these signalling networks, which are often associated with malignant transformation (Vivanco and Sawyers, 2002; Hennessy et al, 2005; Liu et al, 2009; Shackelford and Shaw, 2009; Li et al, 2015). For these reasons, exploration of the signalling cascade generated by these two interconnected pathways may offer new opportunities for exploring the role of cellular metabolism in EOC.

Using laser capture microdissection (LCM) coupled with reverse phase protein microarrays (RPPAs), this study compared the metabolic kinase-driven signalling architecture of early and advanced EOC. Because of their central role in modulating a large panel of metabolic functions (e.g., FFA, glycogen and protein synthesis, and so on), major emphasis was focused on the LKB1AMPK and AKT-mTOR pathways and their downstream substrates. This approach allowed us to shed new light on the biological mechanisms underlying EOC and to explore the role of metabolic kinase signalling in modulating the response to standard adjuvant chemotherapy.

\section{MATERIALS AND METHODS}

Clinical samples. Two independent study sets that included retrospective snap-frozen surgical specimens collected from chemo-naive ovarian cancer patients were provided by the Division of Gynaecology Oncology, University of Brescia, Italy. The discovery set included 72 primary tumours collected between 2001 and 2011 and was used to explore the role of kinase-driven metabolic functions across stages of ovarian cancers. Significant findings were further validated in an independent set of 30 specimens collected between 2003 and 2012 by the same institution. The two study sets were processed independently and printed on a separate set of arrays. Clinicopathological information including FIGO stage, histotype and response to therapy was collected for each patient of the two cohorts. The local institutional review board approved the study protocol (Study Reference Number NP553), and written informed consent was obtained from each patient enrolled in the study.

Sample collection and preparation for RPPA downstream analysis. Samples were frozen in liquid nitrogen within $20 \mathrm{~min}$ upon surgical resection to preserve the integrity of the phosphoproteome. Specimens were then embedded in optimal cutting temperature compound (Sakura Finetek, Torrance, CA, USA), cut into $8 \mu \mathrm{m}$ cryo-sections, mounted on uncharged glass slides, and stored at $-80^{\circ} \mathrm{C}$ until microdissected.

A certified pathologist (LAL) evaluated the amount of tumour present in each specimen using representative haematoxylin-andeosin-stained slides. Immediately before undergoing microdissection, each slide was fixed in $70 \%$ ethanol (Sigma Aldrich, St Louis, MO, USA), washed in deionised water, stained with haematoxylin (Sigma Aldrich) and blued in Scott's Tap Water substitute (Electron Microscopy Sciences, Hatfield, PA, USA), and dehydrated through an ethanol gradient (70, 95, and 100\%) and xylene (Sigma Aldrich). In order to prevent protein degradation, Complete protease inhibitor cocktail tablets (Roche Applied Science, Indianapolis, IN, USA) were added to the ethanol, water, haematoxylin and Scott's Tap Water Substitute (Pin et al, 2014). For each sample an average of 15000 tumour cells were isolated from the surrounding microenvironment using a Pixcell II LCM system (Arcturus, Mountain View, CA, USA).

Microdissected cells were lysed in a 1:1 solution of $2 \mathrm{X}$ TrisGlycine SDS Sample buffer (Invitrogen Life Technologies, Carlsbad, CA, USA) and Tissue Protein Extraction Reagent (Pierce, Rockford, IL, USA) supplemented with $2.5 \%$ of 2-mercaptoethanol (Sigma Aldrich). Cell lysates were boiled for $8 \mathrm{~min}$ and stored at $-80^{\circ} \mathrm{C}$.

Reverse phase protein microarray construction and immunostaining. Using an Aushon 2470 arrayer (Aushon BioSystems, Billerica, MA, USA) equipped with $185 \mu \mathrm{m}$ pins, samples and standard curves for internal quality assurance were printed in triplicate onto Oncyte Avid nitrocellulose-coated slides (Grace Biolabs, Bend, OR, USA) as previously described (Pin et al, 2014). A Sypro Ruby Protein Blot Stain (Molecular Probes, Eugene, OR, USA) protocol was used to stain selected arrays to quantify the total amount of protein within each sample (Pin et al, 2014).

Before immunostaining, each array was first incubated with Reblot Antibody stripping solution (Chemicon, Temecula, CA, USA) for $15 \mathrm{~min}$ at room temperature, followed by two washes in PBS. To minimise potential nonspecific bindings, arrays were then incubated in I-block solution (Tropix, Bedford, MA, USA) for $1 \mathrm{~h}$. Each array was tested with a single primary antibody using an automated system (Dako, Carpinteria, CA, USA). Antibody specificity was tested by immunoblotting using a wide panel of cell lysates as previously described (Signore and Reeder, 2012; Pin et al, 2016). Negative control arrays were incubated with the anti- 
rabbit secondary antibody only to account for unspecific binding and background noise. A commercially available catalysed signal amplification system (Dako) coupled with a biotinylated antirabbit secondary antibody (Vector Laboratories, Inc. Burlingame, CA, USA) and a streptavidin-conjugated IRDye680 (LI-COR Biosciences, Lincoln, NE, USA) were used for the amplification and detection of the fluorescent signal.

Arrays were probed with a total of 117 antibodies targeting protein kinases involved in major cellular functions, and the results of the broad screening were previously published (Sereni et al, 2015). This targeted analysis was limited to the kinases-driven metabolic signalling network of early and advanced EOC with emphasis on 42 proteins contained within the LKB1-AMPK and AKT-mTOR pathways (Supplementary Table 1).

Image acquisition and data analysis. Antibody and Sypro Rubystained arrays were scanned using a laser-based PowerScanner (TECAN, Mönnedorf, Switzerland). Acquired images were analysed using the MicroVigene software version 5.1 (Vigene Tech, Carlisle, MA, USA). This commercially available software performs spot finding, averages the triplicates, subtracts the background from the negative control slide(s), and normalises each sample to the corresponding amount of total protein measured by Sypro Ruby staining. Intra- and inter-assay reproducibility of the RPPA platform has been previously reported (Rapkiewicz et al, 2007; Pierobon et al, 2014).

Statistical analysis. Unsupervised hierarchical clustering analysis using the Ward method was performed in JMP software 5.1.2 (SAS Institute Inc., Cary, NC, USA) to evaluate the overall kinase-driven metabolic signalling across ovarian cancer stages. Because samples were collected between 2002 and 2012, tumours were staged by the enrolling institution using the 1988 FIGO staging system (Zeppernick and Meinhold-Heerlein, 2014). The early-stage group consisted of the stage I-IIB tumours, while the advanced group consisted of stage IIC, III, and IV (Zeppernick and Meinhold-Heerlein, 2014; Prat and FIGO Committee on Gynecologic Oncology, 2015).

To statistically determine whether the metabolic signalling networks varied by tumour stage, the activation level of the 42 proteins selected was compared between early and advanced ovarian cancers. Non-parametric two-sided Wilcoxon rank sum test and one-sided Wilcoxon rank sum test were used for the discovery and the validation sets, respectively. RPPA intensity values were displayed using box and whisker plots created in GraphPad Prism version 6.0 (GraphPad, La Jolla, CA, USA) to capture the median and the minimum and maximum values of each comparison group. Fisher's exact test was performed for categorical variables. $P$-values $\leqslant 0.05$ were considered statistically significant.

\section{RESULTS}

A total of 102 surgical biopsies collected from chemo-naive primary ovarian tumours were used for this analysis (Table 1). In all, 15 of the 72 samples (20.8\%) and 7 of the $30(23.3 \%)$ tumours of the discovery and the validation sets, respectively, were classified as early-stage cancers. The 15 early-stage tumours of the discovery set included the following: endometrioid $(n=7)$; highgrade (G3) serous carcinomas $(n=2)$; clear cell carcinomas $(n=2)$; mucinous carcinomas $(n=2)$; carcinoma of mixed histology $(n=1)$; and undifferentiated carcinomas $(n=1)$. Histotype distributions for early-stage lesions were endometrioid $(n=3)$, clear cell $(n=3)$, and mucinous $(n=1)$ carcinomas for the validation set.

After surgical resection of the primary tumour, 66 of the 72 (91.7\%) patients in the discovery set and 28 of the $30(93.3 \%)$ patients in the validation set received carboplatin-paclitaxel
Table 1. Clinical and pathological characteristics of the 102 patients analysed in the discovery $(n=72)$ and the validation sets $(n=30)$

\begin{tabular}{|c|c|c|}
\hline Characteristics & Discovery set $(n=72)$ & Validation set $(n=30)$ \\
\hline \multirow[t]{2}{*}{$\mathrm{Age}^{\mathbf{a}}$} & $61.5(24-88)$ & $56(36-78)$ \\
\hline & $N(\%)$ & $N(\%)$ \\
\hline $\begin{array}{l}\text { Histology } \\
\text { LGSC }^{\text {b }} \\
\text { HGSC } \\
\text { Endometrioid } \\
\text { Mixed } \\
\text { Clear cell } \\
\text { Mucinous } \\
\text { Undifferentiated }\end{array}$ & $\begin{aligned} & 2(2.8) \\
36 & (50) \\
13 & (18) \\
8 & (11.1) \\
7 & (9.7) \\
3 & (4.2) \\
3 & (4.2)\end{aligned}$ & $\begin{array}{c}1(3.3) \\
15(50) \\
8(26.7) \\
- \\
5(16.7) \\
1(3.3) \\
-\end{array}$ \\
\hline $\begin{array}{l}\text { FIGO stage } \\
\text { Early stage } \\
\text { I } \\
\text { IIA } \\
\text { IIB } \\
\text { Advanced stage } \\
\text { IIC } \\
\text { III } \\
\text { IV }\end{array}$ & $\begin{aligned} 11 & (15.2) \\
2 & (2.8) \\
2 & (2.8) \\
& \\
6 & (8.3) \\
39 & (54.2) \\
12 & (16.7)\end{aligned}$ & $\begin{array}{c}6(20) \\
1(3.3) \\
- \\
3(10) \\
17(56.7) \\
3(10)\end{array}$ \\
\hline $\begin{array}{l}\text { Response to therapy } \\
\text { Resistant }{ }^{c} \\
\text { Sensitive } \\
\text { No CT } \\
\text { N/A }\end{array}$ & $\begin{array}{c}13(18) \\
53(73.6) \\
4(5.6) \\
2(2.8)\end{array}$ & $\begin{aligned} & 5(16.7) \\
& 23(76.6) \\
& 2(6.7) \\
&-\end{aligned}$ \\
\hline \multicolumn{3}{|c|}{$\begin{array}{l}\text { Abbreviations: CT = chemotherapy; FIGO = Fédération Internationale de Gynécologie et } \\
\text { d'Obstétrique; HGSC = high-grade serum carcinoma; LGSC = low-grade serum carcinoma; } \\
\text { N/A = Not Available. } \\
a_{\text {Median and range are reported. }} \\
b_{\text {Both of which were advanced-stage lesions. }} \\
{ }^{c} \text { Patients were classified as platinum-resistant if they developed progression or recurrence of } \\
\text { disease in } \leqslant 6 \text { months from the completion of first-line chemotherapy (Therasse et al, 2000). }\end{array}$} \\
\hline
\end{tabular}

adjuvant chemotherapy. Patients were followed for an average of 56 months after completion of the treatment. Of the 66 patients treated in the discovery set, $13(19.7 \%)$ showed relapse or disease progression within 6 months from the administration of the last treatment and were therefore classified as platinum-resistant. Response to therapy was determined based on CA125 serum levels and RECIST criteria based on conventional imaging techniques (Therasse et al, 2000). In the validation set, 5 of the 28 patients (17.9\%) were classified as resistant to the therapy. All carboplatinpaclitaxel-resistant patients were diagnosed with advanced stages EOC (stage IIC-IV).

The activation of LKB1-AMPK and AKT-mTOR signalling pathways differs between early- and advanced-stage ovarian cancer. Unsupervised hierarchical clustering analysis of kinasedriven metabolic pathways identified two major clusters with distinct metabolic phenotypes (Figure 1). Of interest, stage I and IIA/IIB lesions were contained within the same cluster (14 out of $15 ; 93.3 \%$ ), and were characterised by an overall high activation of the AMPK-AKT-mTOR metabolic signalling network. Advanced-stage ovarian cancers, on the other hand, presented with a more heterogeneous profile. While approximately half of the samples (26 out of $57 ; 45.6 \%$ ) had a systemic activation of the metabolic pathway comparable to the early-stage tumours, 31 of the 32 cases $(96.8 \%)$ contained in the cluster with low activation of the metabolic signature were advanced-stage tumours.

Advanced ovarian cancers are characterised by reduced kinasedriven metabolic activity. To further explore the role of kinase signalling and metabolic activity in ovarian cancers based on stage distribution, a non-parametric mean comparison was performed. Of the 42 proteins measured, 22 were statistically different between 


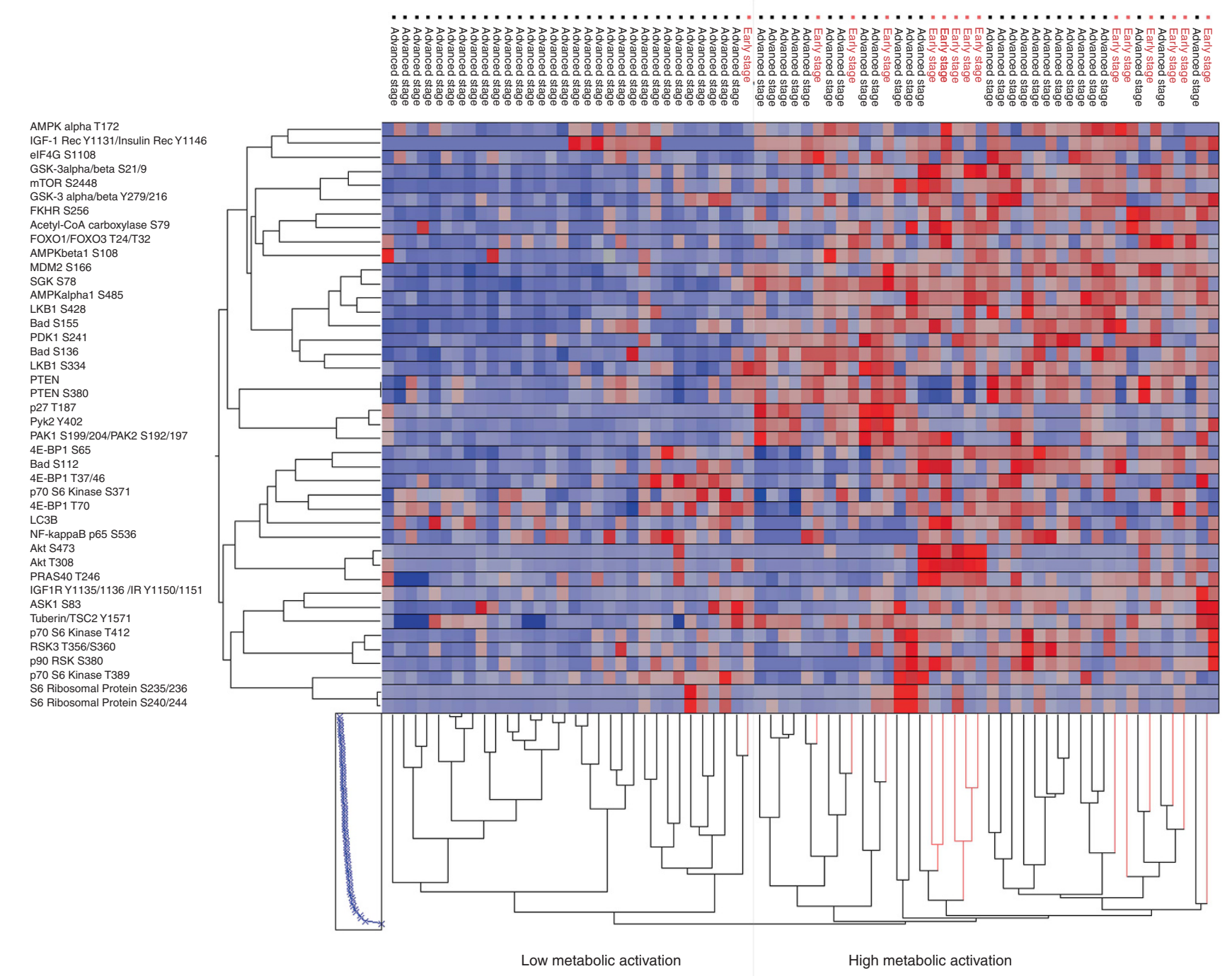

Figure 1. Unsupervised hierarchical clustering analysis of the 42 proteins ( $y$ axis) involved in the energy cellular homeostasis with focus on the LKB1-AMPK and the AKT-mTOR signalling pathways and downstream substrates. The 72 samples included in the discovery set ( $x$ axis) were classified as early (red)- and advanced (black)-stage tumours. The two major clusters (high vs low metabolic activation) were identified by the analysis.

early and advanced tumours (Table 2, panel A). Advanced ovarian tumours presented with an overall downregulation of the LKB1AMPK pathway (LKB1 S428 $P=0.04$, AMPK $\alpha$ T172 $P=0.05$, AMPK $\alpha 1$ S485 $P<0.01$, and AMPK $\beta 1$ S108 $P<0.01)$ and the AKT-mTOR pathway (PDK1 S241 $P=0.02$, AKT S473 $P<0.01$ and AKT T308 $P<0.01$, and mTOR S2448 $P=0.04)$. Deregulation of several interconnected downstream effectors controlling major metabolic activities was also detected in the advanced lesions (Figure 2). Specifically, advanced ovarian cancers were characterised by the following: deregulation of the fatty acid synthesis, including the acetyl-CoA carboxylase (ACC) S79 $(P<0.01)$; decreased protein (p70S6 S371 $P=0.03$ and 4EBP1 S65 $P=0.01$ ) and glycogen (GSK-3 $\alpha / \beta$ S21/9 $P<0.01)$ synthesis; altered redox balance (FOXO1 T24/FOXO3 T32 $P<0.01$ and FOXO1 S256 $P<0.01$ ); altered glucose/amino-acid uptake (IGF-1 Rec Y1131/ Insulin Rec Y1146 $P=0.02)$; and deregulation of the pro-apoptotic and autophagic pathways (BAD S112 $P=0.04$, BAD S155 $P=0.04$, and LC3B $P<0.01$ ).

Similar alterations were also detected in the validation set where several LKB1-AMPK and AKT-mTOR downstream substrates (ACC S79 $P=0.01$, FOXO1 T24/FOXO3 T32 $P<0.01$, FOXO1 S256 $P=0.01$, and GSK-3 $\alpha / \beta \quad$ S21/9 $P<0.01)$ were significantly downregulated in advanced-stage tumours (Table 2, panel B).

Low relative activation of the kinase-driven metabolic signature and phosphorylation of FOXO1/FOXO3 are associated with resistance to carboplatin-paclitaxel adjuvant treatment. Because the AMPK-AKT-mTOR metabolic profile of early-stage ovarian cancers presented a distinctive metabolic profile compared to more advanced tumours, unsupervised hierarchical clustering analysis was repeated for the 66 patients that received adjuvant therapy to explore the role of kinase-driven metabolic changes in the response to conventional carboplatin-paclitaxel adjuvant therapy (Figure 3). To diminish unspecific and heterogeneous signals generated by proteins that were not major contributors in differentiating between early and advanced tumours, the analysis was restricted to proteins that were statistically different.

Of the 66 patients that received adjuvant treatment, 43 were contained within the cluster characterised by overall low activation of the AMPK-AKT-mTOR metabolic signature. Of interest, the proportion of patients with resistant disease was significantly higher among patients with low activation of the metabolic 
Table 2. Proteins that were statistically significant between early- and advanced-stage ovarian cancers in the discovery (panel A) and the validation sets (panel B), respectively

\begin{tabular}{|c|c|c|c|}
\hline & & \multicolumn{2}{|c|}{$\begin{array}{c}\text { RPPA median intensity } \\
\text { values }\end{array}$} \\
\hline Protein & $P$-value & $\begin{array}{l}\text { Early } \\
\text { stages }\end{array}$ & $\begin{array}{l}\text { Advanced } \\
\text { stages }\end{array}$ \\
\hline \multicolumn{4}{|l|}{ Panel A } \\
\hline 4EBP1 S65 & 0.01 & 13095.18 & 8725.46 \\
\hline Acetyl-CoA carboxylase S79 & $<0.01$ & 10625.37 & 4817.45 \\
\hline AKT T308 & $<0.01$ & 4272.69 & 2537.67 \\
\hline AKT S473 & $<0.01$ & 1718.14 & 401.02 \\
\hline AMPK $\alpha$ T172 & 0.05 & 14271.22 & 9302.14 \\
\hline AMPK $\alpha 1$ S485 & $<0.01$ & 10198.54 & 5619.51 \\
\hline AMPK $\beta 1$ S108 & $<0.01$ & 8857.32 & 6627.61 \\
\hline Bad S112 & 0.04 & 2006.20 & 1136.83 \\
\hline Bad S155 & 0.04 & 5089.83 & 4924.61 \\
\hline FOXO1 S256 & $<0.01$ & 12531.49 & 9643.12 \\
\hline FOXO1 T24/FOXO3 T32 & $<0.01$ & 26108.08 & 12444.08 \\
\hline GSK-3 $\alpha / \beta$ S21/9 & $<0.01$ & 16514.60 & 9228.02 \\
\hline IGF-1 RY1135/1136 IR Y1150/1151 & 0.02 & 2472.54 & 1631.08 \\
\hline LC3B & $<0.01$ & 2980.96 & 1271.56 \\
\hline LKB1 S428 & 0.04 & 4895.15 & 3374.49 \\
\hline MDM2 S166 & $<0.01$ & 14559.52 & 9154.49 \\
\hline mTOR S2448 & 0.04 & 12283.35 & 9163.65 \\
\hline p70S6 Kinase S371 & 0.03 & 4167.20 & 3543.96 \\
\hline p90 RSK S380 & 0.03 & 2132.39 & 595.26 \\
\hline PDK1 S241 & 0.02 & 8725.46 & 5721.58 \\
\hline PRAS40 T246 & $<0.01$ & 16139.10 & 11544.91 \\
\hline SGK1 S78 & $<0.01$ & 12569.14 & 9566.29 \\
\hline \multicolumn{4}{|l|}{ Panel B } \\
\hline Acetyl-CoA carboxylase S79 & 0.02 & 11860.86 & 6594.56 \\
\hline AMPK $\alpha 1$ S485 & 0.03 & 38948.66 & 22247.84 \\
\hline FOXO1 S256 & $<0.01$ & 38561.14 & 26903.18 \\
\hline FOXO1 T24/FOXO3 T32 & $<0.01$ & 45251.91 & 28001.12 \\
\hline GSK-3 $\alpha / \beta$ S21/9 & $<0.01$ & 52052.06 & 29143.87 \\
\hline MDM2 S166 & $<0.01$ & 34891.55 & 22247.84 \\
\hline p90 RSK S380 & $<0.01$ & 18676.10 & 29143.87 \\
\hline PRAS40 T246 & 0.03 & 36680.49 & 21829.12 \\
\hline \multicolumn{4}{|c|}{$\begin{array}{l}\text { Abbreviation: RPPA = reverse phase protein microarray. Two-sided non-parametric Wil- } \\
\text { coxon rank sum test was used for the discovery set and a one-sided non-parametric } \\
\text { Wilcoxon rank sum test was used for the validation set. P-value and median RPPA intensity } \\
\text { values for early and advanced stages are reported for each protein. }\end{array}$} \\
\hline
\end{tabular}

signature compared to those tumours that resembled the earlystage lesions (Fisher's exact test $P=0.02$ ). Specifically, 12 of the 13 original samples (92.3\%) classified as resistant to carboplatinpaclitaxel were advanced ovarian cancers that phenotypically presented low activation of the metabolic signature (Figure 3 ). Histotype distribution was found to be homogenous between carboplatin-paclitaxel-sensitive and -resistant tumours (Fisher's exact test $P=0.43$ ). The carboplatin-paclitaxel-resistant group included eight serous carcinomas, two clear cell carcinomas, one carcinoma of mixed histology, and one endometrioid tumour. When the analysis was limited to the end points that reached statistical significance in the validation set, a similar trend was detected with four of the five carboplatin-paclitaxel-resistant patients (80.0\%) with low metabolic activation (Supplementary Figure 1).

Finally, because all carboplatin-paclitaxel-resistant patients were included in the advanced group, changes within a kinasedriven metabolic signalling network of carboplatin-paclitaxelresistant patients were explored in advanced patients. Mean comparison analysis revealed that carboplatin-paclitaxel-resistant lesions have a significant increase in phosphorylation of FOXO1 T24/FOXO3 T32 $(P=0.041$; Figure 4$)$ along with a decrease in activation of 4EBP1 S65 and 4EBP1 T70 $(P=0.004$ and 0.006 , respectively), p70S6 T389 $(P=0.026)$, and LKB1 S334 $(P=0.019)$ compared to the sensitive tumours.

\section{DISCUSSION}

To our knowledge this is the first study correlating kinase-driven metabolic signalling of malignant cells with disease severity in EOC. By using upfront cellular enrichment via LCM these data allow us to obtain a clear picture of the metabolic activity driving tumour epithelium progression while eliminating the confounding signal generated by different cell subpopulations of the tumour microenvironment (e.g., white blood cells, endothelium, nerves, fibroblasts, and so on), which would represent uncontrollable co-varying contributions to the data that could not be subtracted from the overall signalling (Silvestri et al, 2010; Mueller et al, 2014; Baldelli et al, 2015).

In keeping with recent data and postulates indicating that latestage aggressive ovarian cancers arise from the fallopian tube and are aetiologically and biologically entirely different (Kurman, 2013), our functional signalling data indicate that early and advanced EOCs are biochemically distinct and represent more than a stepwise progression within the carcinogenesis process. According to our functional signalling data, early-stage EOCs have an overall higher activation of kinase-driven signalling architecture that regulate energy metabolism. On the other hand, the heterogeneous nature of the signalling architecture of late-stage EOC observed indicates a higher degree of biochemical complexity. The metabolic signalling network of EOC should be further explored as a source of predictive biomarkers of response to conventional chemotherapy (e.g., phosphorylation of FOXO1 T24/ FOXO3 T32) and of novel therapeutic targets. For example, targeting activated LKB1-AMPK signalling pathway or altered autophagy processes may represent new therapeutic modalities for advanced ovarian cancers with low metabolic activity.

Our findings indicate that early-stage EOCs are characterised by a highly activated metabolic signalling architecture involving the LKB1-AMPK energy-regulatory pathway and the AKT-mTOR pro-survival pathway. The activation of the LKB1-AMPK pathway is a recognised compensatory mechanism that early-stage tumours utilise to overcome hypoxia and metabolic stress (Faubert et al, 2013). This compensatory mechanism, commonly described as the Warburg effect, leads to a profound metabolic shift towards aerobic glycolysis, an energy conservation process characterised by increased glucose consumption and activation of catabolic energy production (Vander Heiden et al, 2009; Cairns et al, 2011). A previous study has indicated that $\mathrm{AMPK} \beta$, a modulator of AMPK activation, is overexpressed in early EOC compared to the advanced-stage tumours and that EOC patients with increased AMPK $\alpha$ have more favourable disease-free and overall survival indicating that AMPK may have an important role in ovarian carcinogenesis (Li et al, 2012). Our data confirmed that AMPK signalling is a phenotypic characteristic of early-stage EOC and demonstrates that the activation of AMPK, measured in terms of phosphorylation of AMPK $\alpha$ T172, AMPK $\alpha 1$ S485, and AMPK $\beta 1$ $\mathrm{S} 108$, is increased in early-stage EOC. Moreover, this activation directly affects downstream substrates and specifically ACCmediated fatty acid metabolism and autophagy.

Downregulation of the autophagic marker LC3 and reduced phosphorylation of ACC have been previously described as alterations that characterise solid tumours with less favourable prognosis (Conde et al, 2007; Peracchio et al, 2012; Wang et al, 2013; Valente et al, 2014). Of interest, activation of ACC via dephosphorylation enhances fatty acid synthesis and provides tumours with a readily available energy source known for its role in sustaining tumour growth and progression (Currie et al, 2013; Qu et al, 2016). This change in metabolic signalling may offer a biological explanation for the protective and therapeutic effects of metformin in EOC via increased activation of the AMPK signalling network (Rattan et al, 2011; Irie et al, 2016). 
A

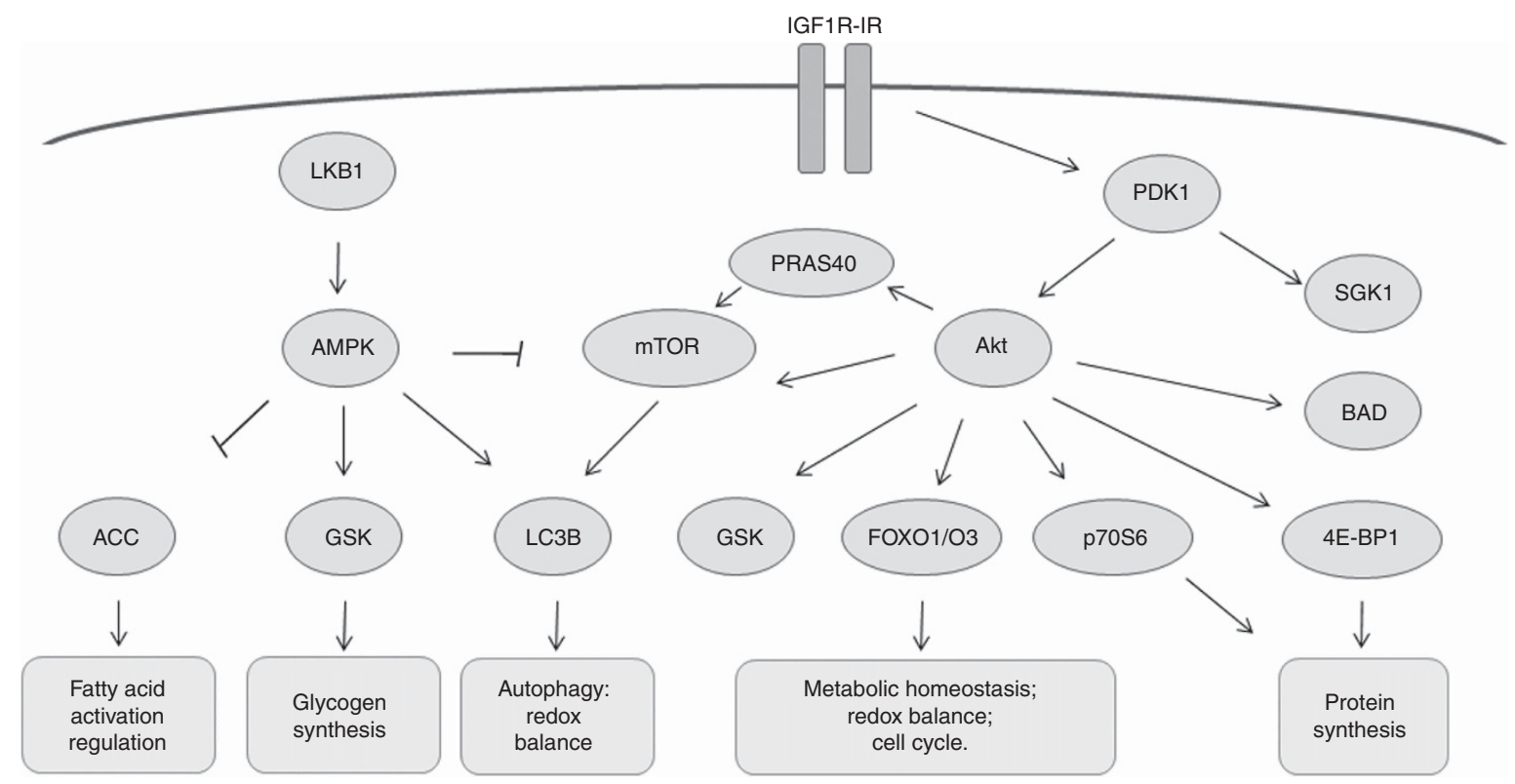

B
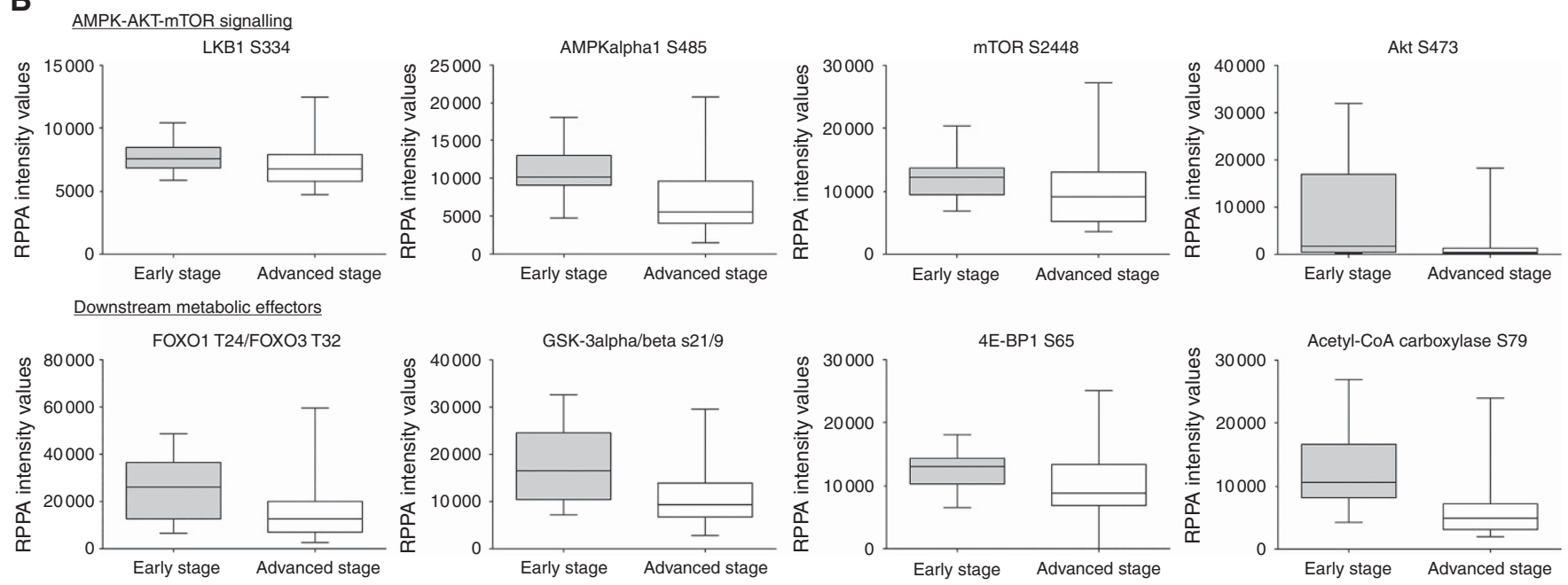

Figure 2. Pathway representation of interconnected proteins that were statistically different between early-and advanced-stage tumours. Members of the LKB1-AMPK and the AKT-mTOR signalling pathways that were highly activated in the early-stage ovarian cancers compared to the advanced stages are represented along with their metabolic functions (A). Box-plots of selected key proteins that reached statistical significance with non-parametric mean comparison illustrate the median and the minimum and maximum values for each group (B).

Our data also indicate that early-stage ovarian cancers are characterised by enhanced activation of the pro-survival AKTmTOR signalling network as characterised by increased phosphorylation of AKT S473 and T308, mTOR S2448, and the downstream substrates p70S6 S371, 4EBP1 S65, GSK-3 $\alpha / \beta$ S21/9, FOXO1 T24/ FOXO3 T32, and FOXO1 S256. While the activation of the LKB1AMPK pathway usually leads to downregulation of the AKT-mTOR signalling pathway, changes in the cellular metabolic needs and the activation of crosstalk between pathways can lead to the activation of the AKT-mTOR pathway in an LKB1-AMPK-independent manner (Elstrom et al, 2004). For example, anaerobic glycolysis and increased glucose metabolisms are known metabolic changes that directly modulate the activation of the AKT-mTOR pathway (Simons et al, 2012). In addition, p90RSK, a protein that was also highly activated in early-stage tumours, can directly modulate mTOR activation via phosphorylation of the tumour suppressor tuberin, further validating that activation of the signalling network is highly complex and driven by a number of compensatory mechanisms (Roux et al, 2004).

Finally, our findings indicate that while advanced ovarian cancers are heterogeneous in terms of their metabolic signalling profile, over $90 \%$ of the carboplatin-paclitaxel-resistant patients have low activation of the metabolic signature along with increased phosphorylation of AKT-mTOR downstream target FOXO1 T24/ FOXO3 T32. This oncosuppressor directly regulates oxidative stress and transcription of several genes involved in cell cycle control, apoptosis, and DNA repair (Eijkelenboom and Burgering, 2013). A previous study exploring the biological mechanisms associated with paclitaxel resistance in ovarian cancer suggested that FOXO1 expression may represent a negative predictor of response to treatment, although this observation was based on a relatively small cohort of patients and the results were only marginally significant (Goto et al, 2008). Our findings further confirm the role of FOXO1 and FOXO3 as potential predictive biomarkers for paclitaxel-based treatment. It has been postulated that an increase in FOXO1 expression may be important in containing oxidative stress and ROS production in paclitaxelresistant EOC. Specifically, overexpression of FOXO1 and its accumulation in the cytosol, a process that is mediated by phosphorylation of the oncosuppressor, may be associated with overexpression of MnSOD, an enzyme directly involved in the 

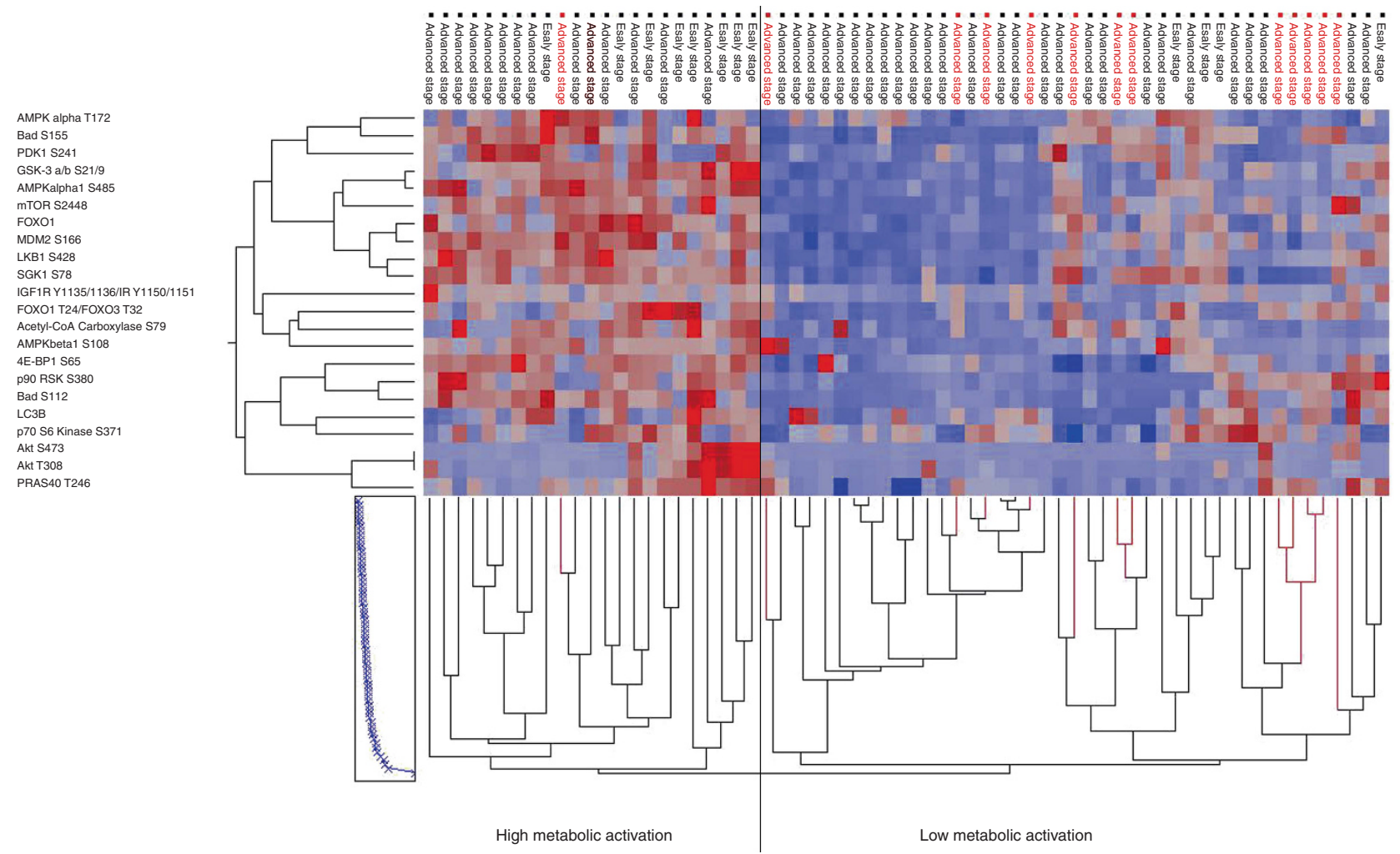

Figure 3. Unsupervised hierarchical clustering analysis of the proteins ( $y$ axis) that reached statistical significance between early and advanced tumours in the discovery set. The analysis was limited to the 66 patients ( $x$ axis) that received carboplatin-paclitaxel adjuvant chemotherapy. Platinum-resistant (red) and platinum-sensitive patients (black) were subdivided based on their metabolic phenotype.

FOX01 T24/FOX03 T32

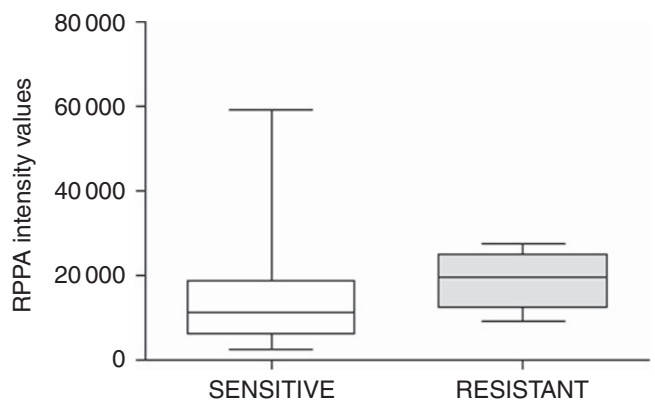

Figure 4. FOXO1 T24/FOXO3 T32 levels in advanced EOC based on response to adjuvant treatment with carboplatin-paclitaxel. Patients that were resistant to adjuvant treatment had significantly higher phosphorylation levels of FOXO1 T24/FOXO3 T32.

oxidative stress response (Goto et al, 2008). Because ROS production can modulate cell growth and response to therapy, the increased expression of MnSOD may prevent ROS accumulation and therefore reduce paclitaxel-associated cytotoxic effects (Goto et al, 2008; Trachootham et al, 2009).

In vitro studies exploring the association between metabolic activity and epithelial-mesenchymal transition (EMT) in cancer cells may offer an additional explanation on the role of FOXO1 and/or FOXO3, and the overall downregulation of the metabolic signature in advanced EOC in terms of response to carboplatinpaclitaxel. It is well known that EMT is a common molecular mechanism used by tumour cells to spread locally and systemically (Thiery, 2002). In vitro studies have indicated that the activation of AMPK via administration of metformin favours the acquisition of an epithelial phenotype (Chou et al, 2014). In keeping with these findings, our analysis revealed that AMPK activation and
E-cadherin expression $(P=0.02)$ were downregulated while $\mathrm{N}$-cadherin was increased (although not significant) in the advanced-stage EOC compared to early-stage lesions (data not shown). Chou and colleagues have shown that the activation of AMPK induces stabilisation and nuclear accumulation of FOXO3a. The nuclear translocation of FOXO3a modulates the transcription of the E-cadherin gene $\mathrm{CDH} 1$ while downregulating genes promoting a mesenchymal-like phenotype. On the basis of this observation, one could speculate that priming carboplatinpaclitaxel-resistant lesions with an AMPK-activating agent such as metformin may pre-sensitise resistant cells to treatment by modulating FOXO1 T24/FOXO3 T32 phosphorylation and cellular localisation. Preclinical studies using in vitro and in vivo models targeting tumours with high FOXO1 T24/FOXO3 T32 levels could be used to test the role of pre-treating EOC resistant tumours with an AMPK-activating agent to promote carboplatin-paclitaxel sensitivity.

Our findings indicate that the metabolic profile of EOC varies greatly based on disease severity with early-stage tumours switching towards anaerobic glycolysis as a compensatory mechanism to overcome the metabolic stress tumour cells undergo. The heterogeneous metabolic signatures of advanced EOC may represent a predictive factor of resistance to conventional chemotherapy and alteration of the LKB1-AMPK signalling pathway along with enhanced autophagy may represent new therapeutic modalities for advanced ovarian cancers with low metabolic activity. The predictive value of this metabolic signature, including phosphorylation of FOXO1 T24/FOXO3 T32, should be further validated prospectively in a clinical trial setting where molecular information is collected along with carboplatinpaclitaxel response.

If our initial findings are prospectively confirmed, the evaluation of the predictive markers here identified could be performed as part of the pathological evaluation of EOC and used to stratify 
patients to adjuvant treatment. In such a setting, patients whose ovarian tumours harbour low relative activation of FOXO1 T24/FOXO3 T32 (or nuclear localisation) would directly receive carboplatin-paclitaxel standard of care, while additional multiomic molecular profiling (including genomic, transcriptomic, and proteomic/phosphoproteomic) may be requested for patients whose ovarian tumours have a high level of FOXO1 T24/FOXO3 T32 to identify actionable targets of individual lesions.

Although genomic alterations are central to tumour development, they may be only partially adequate in capturing compensatory mechanisms that deeply affect drug response, including the establishment of feedback mechanisms, protein pathway activation via secretion of soluble factors by the tumour cells and surrounding microenvironment, or crosstalk between signalling pathways (Pierobon et al, 2017). The addition of proteomic/phosphoproteomic analyses to conventional genomic testing may help identify alterations that are directly driving the malignant lesion. A recent clinical trial targeting refractory metastatic breast cancer has shown that use of a multi-omic molecular profile for treatment selection can be highly beneficial in improving patients' response to treatment (Jameson et al, 2014). The use of stratifying biomarkers such as phosphorylated FOXO1 T24/FOXO3 T32 coupled with a comprehensive multi-omic molecular profiling may offer new therapeutic opportunities for managing patients with advanced EOC more effectively.

\section{ACKNOWLEDGEMENTS}

This work was supported by the College of Science, George Mason University, the Istituto Superiore di Sanità (Programma Italia-USA Oncoproteomica 527/B4/4, Rome, Italy). MIS was a recipient of a PostDoc Fellowship from the University of Brescia, Italy.

\section{CONFLICT OF INTEREST}

The authors are inventors on US Government and Universityassigned patents and patent applications that cover aspects of the technologies discussed such as LCM and RPPAs. As inventors, they are entitled to receive royalties as provided by the US Law and George Mason University policy. LAL, EFP, and MP are consultants to and/or shareholders of Theranostics Health, Inc. EFP and MP are consultants to and/or shareholders of Perthera Inc.

\section{REFERENCES}

Baldelli E, Haura EB, Crinò L, Cress DW, Ludovini V, Schabath MB, Liotta LA, Petricoin EF, Pierobon M (2015) Impact of upfront cellular enrichment by laser capture microdissection on protein and phosphoprotein drug target signaling activation measurements in human lung cancer: Implications for personalized medicine. Proteomics Clin Appl 9(9-10): 928-937.

Cairns RA, Harris IS, Mak TW (2011) Regulation of cancer cell metabolism. Nat Rev Cancer 11(2): 85-95.

Chou CC, Lee KH, Lai IL, Wang D, Mo X, Kulp SK, Shapiro CL, Chen CS (2014) AMPK reverses the mesenchymal phenotype of cancer cells by targeting the Akt-MDM2-Foxo3a signaling axis. Cancer Res 74(17): 4783-4795.

Conde E, Suarez-Gauthier A, García-García E, Lopez-Rios F, Lopez-Encuentra A, García-Lujan R, Morente M, Sanchez-Verde L, Sanchez-Cespedes M (2007) Specific pattern of LKB1 and phospho-acetyl-CoA carboxylase protein immunostaining in human normal tissues and lung carcinomas. Hum Pathol 38(9): 1351-1360.

Currie E, Schulze A, Zechner R, Walther TC, Farese RV (2013) Cellular fatty acid metabolism and cancer. Cell Metab 18(2): 153-161.
Dilokthornsakul P, Chaiyakunapruk N, Termrungruanglert W, Pratoomsoot C, Saokaew S, Sruamsiri R (2013) The effects of metformin on ovarian cancer: a systematic review. Int J Gynecol Cancer 23(9): 1544-1551.

Eijkelenboom A, Burgering BM (2013) FOXOs: signalling integrators for homeostasis maintenance. Nat Rev Mol Cell Biol 14(2): 83-97.

Elstrom RL, Bauer DE, Buzzai M, Karnauskas R, Harris MH, Plas DR, Zhuang H, Cinalli RM, Alavi A, Rudin CM, Thompson CB (2004) Akt stimulates aerobic glycolysis in cancer cells. Cancer Res 64(11): 3892-3899.

Faubert B, Boily G, Izreig S, Griss T, Samborska B, Dong Z, Dupuy F, Chambers C, Fuerth BJ, Viollet B, Mamer OA, Avizonis D, DeBerardinis RJ, Siegel PM, Jones RG (2013) AMPK is a negative regulator of the Warburg effect and suppresses tumor growth in vivo. Cell Metab 17(1): 113-124.

Goto T, Takano M, Hirata J, Tsuda H (2008) The involvement of FOXO1 in cytotoxic stress and drug-resistance induced by paclitaxel in ovarian cancers. Br J Cancer 98(6): 1068-1075.

Groen RS, Gershenson DM, Fader AN (2015) Updates and emerging therapies for rare epithelial ovarian cancers: one size no longer fits all. Gynecol Oncol 136(2): 373-383.

Hanahan D, Weinberg RA (2011) Hallmarks of cancer: the next generation. Cell 144(5): 646-674.

Hennessy BT, Smith DL, Ram PT, Lu Y, Mills GB (2005) Exploiting the PI3K/AKT pathway for cancer drug discovery. Nat Rev Drug Discov 4(12): 988-1004.

Herzog TJ, Spetzler D, Xiao N, Burnett K, Maney T, Voss A, Reddy S, Burger R, Krivak T, Powell M, Friedlander M, McGuire W (2016) Impact of molecular profiling on overall survival of patients with advanced ovarian cancer. Oncotarget 7(15): 19840-19849.

Irie H, Banno K, Yanokura M, Iida M, Adachi M, Nakamura K, Umene K, Nogami Y, Masuda K, Kobayashi Y, Tominaga E, Aoki D (2016) Metformin: a candidate for the treatment of gynecological tumors based on drug repositioning. Oncol Lett 11(2): 1287-1293.

Jameson GS, Petricoin EF, Sachdev J, Liotta LA, Loesch DM, Anthony SP, Chadha MK, Wulfkuhle JD, Gallagher RI, Reeder KA, Pierobon M, Fulk MR, Cantafio NA, Dunetz B, Mikrut WD, Von Hoff DD, Robert NJ (2014) A pilot study utilizing multi-omic molecular profiling to find potential targets and select individualized treatments for patients with previously treated metastatic breast cancer. Breast Cancer Res Treat 147(3): 579-588.

Kumar S, Meuter A, Thapa P, Langstraat C, Giri S, Chien J, Rattan R, Cliby W, Shridhar V (2013) Metformin intake is associated with better survival in ovarian cancer: a case-control study. Cancer 119(3): 555-562.

Kurman RJ (2013) Origin and molecular pathogenesis of ovarian high-grade serous carcinoma. Ann Oncol 24(Suppl 10): 16-21.

Kurman RJ, Shih IM (2010) The origin and pathogenesis of epithelial ovarian cancer: a proposed unifying theory. Am J Surg Pathol 34(3): 433-443.

Kurman RJ, Shih IM (2011) Molecular pathogenesis and extraovarian origin of epithelial ovarian cancer - shifting the paradigm. Hum Pathol 42(7): 918-931.

Li C, Liu VW, Chiu PM, Chan DW, Ngan HY (2012) Over-expressions of AMPK subunits in ovarian carcinomas with significant clinical implications. BMC Cancer 12: 357.

Li W, Saud SM, Young MR, Chen G, Hua B (2015) Targeting AMPK for cancer prevention and treatment. Oncotarget 6(10): 7365-7378.

Liu P, Cheng H, Roberts TM, Zhao JJ (2009) Targeting the phosphoinositide 3-kinase pathway in cancer. Nat Rev Drug Discov 8(8): 627-644.

Mueller C, deCarvalho AC, Mikkelsen T, Lehman NL, Calvert V, Espina V, Liotta LA, Petricoin EF (2014) Glioblastoma cell enrichment is critical for analysis of phosphorylated drug targets and proteomic-genomic correlations. Cancer Res 74(3): 818-828.

Nelson MA, Radmacher MD, Simon R, Aickin M, Yang J, Panda L, Emerson J, Roe D, Adair L, Thompson F, Bangert J, Leong SP, Taetle R, Salmon S, Trent J (2000) Chromosome abnormalities in malignant melanoma: clinical significance of nonrandom chromosome abnormalities in 206 cases. Cancer Genet Cytogenet 122(2): 101-109.

Peracchio C, Alabiso O, Valente G, Isidoro C (2012) Involvement of autophagy in ovarian cancer: a working hypothesis. J Ovarian Res 5(1): 22.

Pierobon M, Ramos C, Wong S, Hodge KA, Aldrich J, Byron SA, Anthony SP, Robert NJ, Northfelt DW, Jahanzeb M, Vocila L, Wulfkuhle JD, Gambara G, Gallagher RI, Dunetz B, Hoke N, Dong T, Craig DW, Cristofanilli M, Leyland-Jones M, Liotta LA, O'Shaughnessy JA, Carpten JD, Petricoin EF (2017) Enrichment of PIK3-AKT-MTOR 
pathway activation in hepatic metastases from breast cancer. Clin Cancer Res; e-pub ahead of print 26 April 2017; doi:10.1158/1078-0432.CCR-162656.

Pierobon M, Silvestri A, Spira A, Reeder A, Pin E, Banks S, Parasido E, Edmiston K, Liotta L, Petricoin E (2014) Pilot phase I/II personalized therapy trial for metastatic colorectal cancer: evaluating the feasibility of protein pathway activation mapping for stratifying patients to therapy with imatinib and panitumumab. J Proteome Res 13(6): 2846-2855.

Pin E, Federici G, Petricoin EF (2014) Preparation and use of reverse protein microarrays. Curr Protoc Protein Sci 75: Unit 27.7.

Pin E, Stratton S, Belluco C, Liotta L, Nagle R, Hodge KA, Deng J, Dong T, Baldelli E, Petricoin E, Pierobon M (2016) A pilot study exploring the molecular architecture of the tumor microenvironment in human prostate cancer using laser capture microdissection and reverse phase protein microarray. Mol Oncol 10(10): 1585-1594.

Prat J, FIGO Committee on Gynecologic Oncology (2015) FIGO's staging classification for cancer of the ovary, fallopian tube, and peritoneum: abridged republication. J Gynecol Oncol 26(2): 87-89.

Qu Q, Zeng F, Liu X, Wang QJ, Deng F (2016) Fatty acid oxidation and carnitine palmitoyltransferase I: emerging therapeutic targets in cancer. Cell Death Dis 7: e2226.

Rapkiewicz A, Espina V, Zujewski JA, Lebowitz PF, Filie A, Wulfkuhle J, Camphausen K, Petricoin EF, Liotta LA, Abati A (2007) The needle in the haystack: application of breast fine-needle aspirate samples to quantitative protein microarray technology. Cancer 111(3): 173-184.

Rattan R, Giri S, Hartmann LC, Shridhar V (2011) Metformin attenuates ovarian cancer cell growth in an AMP-kinase dispensable manner. J Cell Mol Med 15(1): 166-178.

Reade CJ, McVey RM, Tone AA, Finlayson SJ, McAlpine JN, Fung-Kee-Fung M, Ferguson SE (2014) The fallopian tube as the origin of high grade serous ovarian cancer: review of a paradigm shift. J Obstet Gynaecol Can 36(2): 133-140.

Rosendahl M, Høgdall CK, Mosgaard BJ (2016) Restaging and Survival Analysis of 4036 Ovarian Cancer Patients According to the 2013 FIGO Classification for Ovarian, Fallopian Tube, and Primary Peritoneal Cancer. Int J Gynecol Cancer 26(4): 680-687.

Roux PP, Ballif BA, Anjum R, Gygi SP, Blenis J (2004) Tumor-promoting phorbol esters and activated Ras inactivate the tuberous sclerosis tumor suppressor complex via p90 ribosomal S6 kinase. Proc Natl Acad Sci USA 101(37): 13489-13494.

Sereni MI, Baldelli E, Gambara G, Deng J, Zanotti L, Bandiera E, Bignotti E, Ragnoli M, Tognon G, Ravaggi A, Meani F, Memo M, Angioli R, Liotta LA, Pecorelli SL, Petricoin E, Pierobon M (2015) Functional characterization of epithelial ovarian cancer histotypes by drug target based protein signaling activation mapping: implications for personalized cancer therapy. Proteomics 15(2-3): 365-373.

Shackelford DB, Shaw RJ (2009) The LKB1-AMPK pathway: metabolism and growth control in tumour suppression. Nat Rev Cancer 9(8): 563-575.

Siegel RL, Miller KD, Jemal A (2016) Cancer statistics, 2016. CA Cancer J Clin 66(1): 7-30.
Signore M, Reeder KA (2012) Antibody validation by western blotting. Methods Mol Biol 823: 139-155.

Silvestri A, Colombatti A, Calvert VS, Deng J, Mammano E, Belluco C, De Marchi F, Nitti D, Liotta LA, Petricoin EF, Pierobon M (2010) Protein pathway biomarker analysis of human cancer reveals requirement for upfront cellular-enrichment processing. Lab Invest 90(5): 787-796.

Simons AL, Orcutt KP, Madsen JM, Scarbrough SDR (2012) The role of Akt pathway signaling in glucose metabolism and metabolic oxidative stress. In: DR Spitz, KJ Dornfeld, K Krishnan, D Gius (eds). Oxidative Stress in Cancer Biology and Therapy. Humana Press, Springer Nature: New York, NY, USA, pp 21-46.

Taetle R, Aickin M, Yang JM, Panda L, Emerson J, Roe D, Adair L, Thompson F, Liu Y, Wisner L, Davis JR, Trent J, Alberts DS (1999) Chromosome abnormalities in ovarian adenocarcinoma: I. Nonrandom chromosome abnormalities from 244 cases. Genes Chromosomes Cancer 25(3): 290-300.

Therasse P, Arbuck SG, Eisenhauer EA, Wanders J, Kaplan RS, Rubinstein L, Verweij J, Van Glabbeke M, van Oosterom AT, Christian MC, Gwyther SG (2000) New guidelines to evaluate the response to treatment in solid tumors. European Organization for Research and Treatment of Cancer, National Cancer Institute of the United States, National Cancer Institute of Canada. J Natl Cancer Inst 92(3): 205-216.

Thiery JP (2002) Epithelial-mesenchymal transitions in tumour progression. Nat Rev Cancer 2(6): 442-454.

Trachootham D, Alexandre J, Huang P (2009) Targeting cancer cells by ROSmediated mechanisms: a radical therapeutic approach? Nat Rev Drug Discov 8(7): 579-591.

Valente G, Morani F, Nicotra G, Fusco N, Peracchio C, Titone R, Alabiso O, Arisio R, Katsaros D, Benedetto C, Isidoro C (2014) Expression and clinical significance of the autophagy proteins BECLIN 1 and LC3 in ovarian cancer. Biomed Res Int 2014: 462658.

Vander Heiden MG, Cantley LC, Thompson CB (2009) Understanding the Warburg effect: the metabolic requirements of cell proliferation. Science 324(5930): 1029-1033.

Vivanco I, Sawyers CL (2002) The phosphatidylinositol 3-Kinase AKT pathway in human cancer. Nat Rev Cancer 2(7): 489-501.

Wang J, Pan XL, Ding LJ, Liu DY, Da-Peng Lei, Jin T (2013) Aberrant expression of Beclin-1 and LC3 correlates with poor prognosis of human hypopharyngeal squamous cell carcinoma. PLOS ONE 8(7): e69038.

Zeppernick F, Meinhold-Heerlein I (2014) The new FIGO staging system for ovarian, fallopian tube, and primary peritoneal cancer. Arch Gynecol Obstet 290(5): 839-842.

This work is published under the standard license to publish agreement. After 12 months the work will become freely available and the license terms will switch to a Creative Commons AttributionNonCommercial-Share Alike 4.0 Unported License.

Supplementary Information accompanies this paper on British Journal of Cancer website (http://www.nature.com/bjc) 